tions existantes est épuisée, et des propriétés spéciales se sont développées pour s'adapter à ces conditions; ou bien il s'est produit un changement avantageux et l'individu transformé surpasse le type original. Tout bactériologiste doit être certain que les bactéries, aussi bien que les formes de vie plus élevées, sont gouvernées par les lois biologiques.

\title{
QUELQUES REMARQUES SUR L'ÉTUDE DE CH. BARTHEL : INFLUENCE DES MOISISSURES SUR LES FERMENTS LACTIQUES (1)
}

\author{
Par le Dr JAROSLAV DVORAK
}

Dans le travail de Ch. Banthes, celui-ci a trouvé que la présence des moisissures dans un milieu nutritif exerce une influence favorable sur les bactéries lactiques, parce que les matières produites par celles-là ont un effet stimulateur sur celles-ci.

Se basant sur toute une série d'essais, cet auteur émet la supposition que l'influence incontestablement favorable des moissisures sur les bactéries lactiques peut avoir certaine importance pratique dans la fromagerie, où les deux groupes de micro-organismes se développent simultanément dans un milieu naturel, comme cela est dans la fabrication des fromages de Roquefort, de Gorgonzola, etc.

Déjà, pendant les années 1915-1917, j’ai fait des expériences à l'Institut lactologique de l'Ecole polytechnique à Prague, où j'étudiais l'influence qu'exercent les bactéries lactiques sur la croissance des moisissures utiles pour fromagerie du genre de Penicillium et j'ai obtenu des résultats analogues à ceux de Ch. Barthel.

J'ai publié l'ensemble de ces études dans l'ouvrage : Etudes biochimiques de quelques moisissures du genre Penicillium, importantes en fromagerie (Rozprasy ceské akademie ped a umeni, classe II, $\left.n^{0} 31,1917\right)$. C'est le professeur Laxa qui en a rapporté le résumé dans la revue le Lait, $1^{\text {re }}$ année, p. 361, 1920.

Puisque l'auteur ne cite pas mon travail, je crois de mon devoir d'attirer, moi-même l'attention sur les conclusions que j'y ai formulées.

A part une quantité d'autres observations, j'ai étudié l'influence symbiotique des bactéries lactiques et des moisissures sur la peptonisation de la caséine en présence du lactose dans un milieu neutre et dans le lait. Dans mon travail, à la page 20 , je dis ce qui suit : "Les bactéries lactiques, par leur formation d'acide lactique, favo-

(1) Le Lait, $4^{\mathrm{e}}$ année, $\mathrm{n}^{\circ} 39$. 
risent la végétation des moisissures ; la décomposition de la caséine est profonde, il est vrai, mais la formation des acides volatils est fortement ou complètement limitée.

“ Le Penicillium Roqueforti donne dans ce eas une odeur typique du fromage de Roquefort, et comme cela n'a pas été observé dans la culture avec la caséine en milieu acide, il faut considérer l'influence symbiotique des bactéries lactiques comme condition prineipale de la naissance de l'odeur si appréciée dans le fromage de Roquefort.

“ En comparant les résultats présents avec ceux qu'on avait obtenus sur la easéine avec l'acide lactique, on voit que le Penicillium Roqueforti prospère le mieux en présence des bactéries lactiques et du lactose. "

A la page 32 , je dis ce qui suit: "Mes expériences effectuées avec la caséine pure et la culture pure du Penicillium Roqueforti ont complété les observations dans le sens que la formation de l'odeur s'effectue même dans la simple caséine en présence des bactéries lactiques, donc même sans la 'graisse du lait et sans l'action de l'Oidium lactis. Il est évident que la graisse du lait contribue à la formation de l'odeur et, en sa présence, on obtient des cultures d'une odeur plus prononcée que celle qu'on obtient dans la caséine pure. 》)

J'ai trouvé qu'il était nécessaire de compléter, par les présentes remarques, les expériences de BARTHEL, intéressantes et préeieuses, ainsi que les déductions qui en ressortent et qui ont une valeur pour moi d'autant plus grande qu'elles confirment mes observations dans toute leur étendue.

\title{
CE QUE DOIT SAVOIR UN BON CONTRÓLEUR LAITIER ET BEURRIER
}

\author{
par A.-M. LEROY \\ Ingénieur agronome, \\ Chef de travaux à l'Institut National Agronomique.
}

- surre -

\section{(f) Ineluence sur la lactation de certaines substances ou de CERTAINS GROUPES DE SUBSTANCES ALIMENTAIRES.}

Le problème des " actions spécifiques " de certaines substances alimentaires sur la mamelle a donné lieu à des controverses souvent passionnées. De nombreux praticiens attachent une importance, peut-être excessive, à quelques fourrages particuliers, dont ils admettent à priori la suprématie sur tous les autres; mais leur avis est loin d'être partagé par tous. Entraînés par l'intérêt de la question, 